\title{
Monte Carlo simulations of the $\mathbf{n}_{-}$TOF lead spallation target with the Geant4 toolkit: A benchmark study
}

J. Lerendegui-Marco ${ }^{1, a}$, M.A. Cortés-Giraldo ${ }^{1}$, C. Guerrero ${ }^{1}$, J.M. Quesada ${ }^{1}$, S. Lo Meo ${ }^{2,3}$, C. Massimi ${ }^{3,4}$, M. Barbagallo ${ }^{5}$, N. Colonna ${ }^{5}$, D. Mancussi ${ }^{6}$, F. Mingrone ${ }^{7}$, M. Sabaté-Gilarte ${ }^{7,1}$, G. Vannini ${ }^{3,4}$, V. Vlachoudis ${ }^{7}$, O. Aberle ${ }^{7}$, J. Andrzejewski ${ }^{8}$, L. Audouin ${ }^{9}$, M. Bacak ${ }^{6,7,10}$, J. Balibrea ${ }^{11}$, F. Bečváŕr ${ }^{12}$, E. Berthoumieux ${ }^{6}$, J. Billowes ${ }^{13}$, D. Bosnar ${ }^{14}$, A. Brown ${ }^{15}$, M. Caamaño ${ }^{16}$, F. Calviño ${ }^{17}$, M. Calviani ${ }^{7}$, D. Cano-Ott ${ }^{11}$, R. Cardella ${ }^{7}$, A. Casanovas ${ }^{17}$, F. Cerutti ${ }^{7}$, Y.H. Chen ${ }^{9}$, E. Chiaveri ${ }^{1,7,13}$, G. Cortés ${ }^{17}$, L. Cosentino ${ }^{18}$, L.A. Damone ${ }^{5,19}$, M. Diakaki ${ }^{6}$, C. Domingo-Pardo ${ }^{20}$, R. Dressler ${ }^{21}$, E. Dupont ${ }^{6}$, I. Durán ${ }^{16}$, B. Fernández-Domínguez ${ }^{16}$, A. Ferrari ${ }^{7}$, P. Ferreira ${ }^{22}$, P. Finocchiaro ${ }^{18}$, K. Göbel ${ }^{23}$, M.B. Gómez-Hornillos ${ }^{18}$, A.R. García ${ }^{11}$, A. Gawlik ${ }^{8}$, S. Gilardoni ${ }^{7}$, T. Glodariu ${ }^{24}$, I.F. Gonçalves ${ }^{22}$, E. González ${ }^{11}$, E. Griesmayer ${ }^{10}$, F. Gunsing ${ }^{6,7}$, H. Harada ${ }^{25}$, S. Heinitz ${ }^{21}$, J. Heyse ${ }^{26}$, D.G. Jenkins ${ }^{15}$, E. Jericha ${ }^{10}$, F. Käppeler ${ }^{27}$, Y. Kadi ${ }^{7}$, A. Kalamara ${ }^{28}$, P. Kavrigin ${ }^{10}$, A. Kimura ${ }^{25}$, N. Kivel ${ }^{21}$, M. Kokkoris ${ }^{28}$, M. Krtička ${ }^{12}$, D. Kurtulgil ${ }^{23}$, E. Leal-Cidoncha ${ }^{16}$, C. Lederer ${ }^{29}$, H. Leeb ${ }^{10}$, S.J. Lonsdale ${ }^{29}$, D. Macina ${ }^{7}$, J. Marganiec ${ }^{8,30}$, T. Martínez ${ }^{11}$, A. Masi ${ }^{7}$, P. Mastinu ${ }^{31}$, M. Mastromarco ${ }^{5}$, E.A. Maugeri ${ }^{21}$, A. Mazzone ${ }^{5,32}$, E. Mendoza ${ }^{11}$, A. Mengoni ${ }^{27}$, P.M. Milazzo ${ }^{33}$, A. Musumarra ${ }^{18,34}$, A. Negret ${ }^{24}$, R. Nolte ${ }^{30}$, A. Oprea ${ }^{24}$, N. Patronis ${ }^{35}$, A. Pavlik ${ }^{36}$, J. Perkowski ${ }^{8}$, I. Porras ${ }^{37}$, J. Praena ${ }^{37}$, D. Radeck ${ }^{30}$, T. Rauscher ${ }^{38,39}$, R. Reifarth ${ }^{23}$, P.C. Rout ${ }^{40}$, C. Rubbia ${ }^{7}$, J.A. Ryan ${ }^{13}$, A. Saxena ${ }^{40}$, P. Schillebeeckx ${ }^{26}$, D. Schumann ${ }^{21}$, A.G. Smith ${ }^{13}$, N.V. Sosnin ${ }^{13}$, A. Stamatopoulos ${ }^{28}$, G. Tagliente ${ }^{5}$, J.L. Tain ${ }^{20}$, A. Tarifeño-Saldivia ${ }^{13}$, L. Tassan-Got ${ }^{9}$, S. Valenta ${ }^{12}$, V. Variale ${ }^{5}$, P. Vaz ${ }^{22}$, A. Ventura ${ }^{28}$, R. Vlastou ${ }^{28}$, A. Wallner ${ }^{41}$, S. Warren ${ }^{13}$, P.J. Woods ${ }^{29}$, T. Wright ${ }^{13}$, P. Žugec ${ }^{14,7}$, and the n_TOF Collaboration

1 Universidad de Sevilla, Spain

2 Agenzia nazionale per le nuove tecnologie (ENEA), Bologna, Italy

3 Istituto Nazionale di Fisica Nucleare, Sezione di Bologna, Italy

${ }^{4}$ Dipartimento di Fisica e Astronomia, Università di Bologna, Italy

5 Istituto Nazionale di Fisica Nucleare, Sezione di Bari, Italy

${ }^{6}$ CEA Saclay, Irfu, Gif-sur-Yvette, France

${ }^{7}$ European Organization for Nuclear Research (CERN), Switzerland

${ }^{8}$ University of Lodz, Poland

9 Institut de Physique Nucléaire, CNRS-IN2P3, Univ. Paris-Sud, Université Paris-Saclay, 91406 Orsay Cedex, France

10 Technische Universität Wien, Austria

11 Centro de Investigaciones Energeticas Medioambientales y Tecnológicas (CIEMAT), Spain

12 Charles University, Prague, Czech Republic

13 University of Manchester, UK

14 University of Zagreb, Croatia

15 University of York, UK

16 University of Santiago de Compostela, Spain

17 Universitat Politècnica de Catalunya, Spain

18 INFN Laboratori Nazionali del Sud, Catania, Italy

19 Dipartimento di Fisica, Università degli Studi di Bari, Italy

${ }^{20}$ Instituto de Física Corpuscular, Universidad de Valencia, Spain

21 Paul Scherrer Institut (PSI), Villingen, Switzerland

22 Instituto Superior Técnico, Lisbon, Portugal

23 Goethe University Frankfurt, Germany

${ }^{24}$ Horia Hulubei National Institute of Physics and Nuclear Engineering, Romania

25 Japan Atomic Energy Agency (JAEA), Tokai-mura, Japan

26 European Commission, Joint Research Centre, Geel, Retieseweg 111, 2440 Geel, Belgium

27 Karlsruhe Institute of Technology, Campus North, IKP, 76021 Karlsruhe, Germany

28 National Technical University of Athens, Greece

29 School of Physics and Astronomy, University of Edinburgh, UK

30 Physikalisch-Technische Bundesanstalt (PTB), Bundesallee 100, 38116 Braunschweig, Germany

31 Istituto Nazionale di Fisica Nucleare, Sezione di Legnaro, Italy

32 Consiglio Nazionale delle Ricerche, Bari, Italy

33 Istituto Nazionale di Fisica Nucleare, Sezione di Trieste, Italy

a e-mail: jlerendegui@us.es

(C) The Authors, published by EDP Sciences. This is an Open Access article distributed under the terms of the Creative Commons Attribution License 4.0 (http://creativecommons.org/licenses/by/4.0/). 
34 Dipartimento di Fisica e Astronomia, Università di Catania, Italy

35 University of Ioannina, Greece

36 University of Vienna, Faculty of Physics, Vienna, Austria

37 University of Granada, Spain

38 Department of Physics, University of Basel, Switzerland

39 Centre for Astrophysics Research, University of Hertfordshire, UK

40 Bhabha Atomic Research Centre (BARC), India

41 Australian National University, Canberra, Australia

\begin{abstract}
Monte Carlo (MC) simulations are an essential tool to determine fundamental features of a neutron beam, such as the neutron flux or the $\gamma$-ray background, that sometimes can not be measured or at least not in every position or energy range. Until recently, the most widely used MC codes in this field had been MCNPX and FLUKA. However, the Geant 4 toolkit has also become a competitive code for the transport of neutrons after the development of the native Geant 4 format for neutron data libraries, G4NDL. In this context, we present the Geant 4 simulations of the neutron spallation target of the $\mathrm{n}_{-}$TOF facility at CERN, done with version 10.1 .1 of the toolkit. The first goal was the validation of the intra-nuclear cascade models implemented in the code using, as benchmark, the characteristics of the neutron beam measured at the first experimental area (EAR1), especially the neutron flux and energy distribution, and the time distribution of neutrons of equal kinetic energy, the so-called Resolution Function. The second goal was the development of a Monte Carlo tool aimed to provide useful calculations for both the analysis and planning of the upcoming measurements at the new experimental area (EAR2) of the facility.
\end{abstract}

\section{Introduction and motivation}

The neutron beam of the $\mathrm{n}_{-}$TOF facility is generated through spallation reactions produced by $20 \mathrm{GeV} / \mathrm{c}$ protons provided by the CERN Proton Synchrotron (PS) on a $40 \mathrm{~cm}$ thick lead target. The neutrons produced inside the lead target are partially moderated and travel towards the two experimental areas along two different beam lines: a $185 \mathrm{~m}$ vacuum pipe oriented in the forward direction to the experimental area \#1 (EAR1) [1], and a $19 \mathrm{~m}$ one oriented vertically towards the experimental area \#2 (EAR2) [2].

Monte Carlo (MC) simulations are an essential tool to determine fundamental features of a neutron beam, such as the neutron flux or the $\gamma$-ray background, that sometimes can not be measured or at least not in every position or energy range. Indeed, in the case of time-of-flight facilities, the energy resolution broadening, known as the Resolution Function, can not be measured and must be extracted from MC simulations. Until recently, the most widely used MC codes had been MCNP [3] and FLUKA [4]. However the Geant4 toolkit $[5,6]$ has become a competitive code also in this field, especially after the work of Mendoza et al. [7] to adapt the evaluated neutron libraries to Geant 4 native format, G4NDL.

\section{Geant4 simulations: Geometry, scoring and physics lists}

The main component of the $\mathrm{n}_{-} \mathrm{TOF}$ spallation target assembly is a $60 \mathrm{~cm}$ diameter and $40 \mathrm{~cm}$ thick lead core. Neutrons escaping this lead cylinder pass through several moderating layers $\left(\mathrm{H}_{2} \mathrm{O}\right.$ and borated $\left.\mathrm{H}_{2} \mathrm{O}\right)$ expanding the energy range from $\mathrm{GeV}$ to thermal. An illustrative view of the target-moderator assembly as implemented in Geant4 is presented in Fig. 1, including not only the lead core and water moderators but all the surrounding materials, that also play a role in the energy distribution of the neutron flux, the $\gamma$-ray spectrum, the beam profile or the Resolution Function. A more detailed description of the target geometry and the exits towards both experimental areas can be found in Refs. [8,9].
In our simulations the $20 \mathrm{GeV} / \mathrm{c}$ proton beam impinges on the target in the direction shown in Fig. 1 with a polar angle of 10 degrees with respect to the target central axis, producing hundreds of neutrons and $\gamma$-rays per proton, emitted in all directions. Secondary particles produced in the target are followed and tracked up to the scoring volumes positioned at the entrance of the vacuum beam pipes where their position, time, angle, energy and particle type are registered. These scoring volumes shown in Fig. 1, are the end point of our MC simulation.

Geant4 v10.1.1 provides a wide variety of physics models that apply in different energy regimes. To perform these simulations we worked with the officially released Physics Lists (PL) that include either the Fritiof (FTF) model or the Quark-Gluon-String ( $Q G S)$ model above $\sim 10 \mathrm{GeV}$; the $P$ after $F T F$ or $Q G S$ denotes that the Geant 4 native Precompound model is taken into account for nuclear de-excitation. Below $\sim 10 \mathrm{GeV}$ three different intranuclear de-excitation cascade models are available: INCLXX [10,11], Bertini (BERT) [12] or Binary Cascade
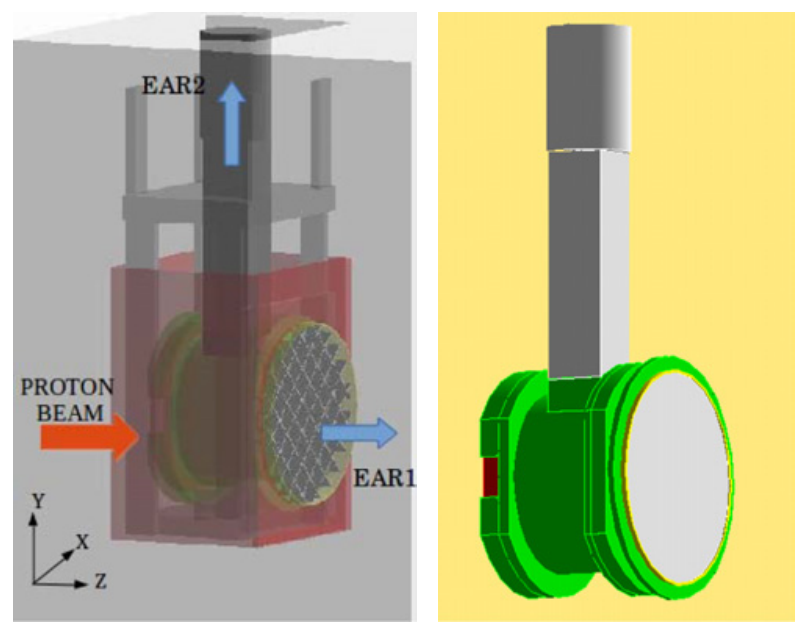

Figure 1. Left: detailed geometry model of the spallation target assembly as implemented in Geant4. Right: MC simulation finishes when particles exiting the target are registered in the scoring volumes highlighted in a lighter color. 
(BIC) [13]. Neutron induced reactions below $20 \mathrm{MeV}$ are simulated by means of the G4NeutronHP package, using the ENDF/B-VII.0 cross section library [11] in our case. Last, we have also considered the Geant 4 built-in special treatment of the Thermal Scattering of neutrons below $4 \mathrm{eV}$. This is not a feature included in any official Geant 4 physics list. Thus, to make this clearer we denote our physics lists using $H P T$, instead of the official notation $H P$ used for the G4NeutronHP package.

\section{Simplified optical transport to the EARs}

The Geant 4 simulation ends when the neutrons or photons entering the scoring volumes (highlighted in white in Fig. 1) are registered into a text file. We only accepted particles with incidence angle at the scoring surface smaller than $4 \mathrm{deg}$, since the energy distribution within this angle interval is isotropic for neutron energies below $1 \mathrm{GeV}$, as has been proven in Refs. [8,9]. Our final results should describe the neutron beam features at the experimental areas; however, detailed MC simulations of the full beamline would be unaffordable concerning CPU time [9]. To solve this issue we have used a code that reduces the transport along the beam line to a problem of beam optics. In brief, a large number of particles is emitted for each particle stored in the Monte Carlo output file, conserving the original energy, time and position at the scorer, but varying the direction isotropically within a cone aperture calculated according to the angular acceptance of the first collimator. The position and dimensions of the collimators are included in this code that determines whether a particle reaches the EAR or hits one of the collimators, being thus rejected.

\section{EAR1: Benchmarking Geant4}

The fast development of the Geant4 [5] simulation toolkit and the work done by Mendoza et al. [7] to include evaluated cross section libraries in Geant4 have recently made it possible to rely on this toolkit for the simulation of the neutron transport. As a first step we wanted to benchmark Geant4 for the specific case of spallation neutrons, trying to reproduce the absolute value and energy dependence of the experimental neutron flux in $\mathrm{n}_{-}$TOF-EAR1 [14]. The energy dependence of the average neutron flux per pulse, considering the nominal proton pulse intensity of $7 \cdot 10^{12}$ protons, has been calculated from the output of the Geant4 simulation after the geometrical transport to EAR1. The upper panel of Fig. 2 compares all the studied Geant4 Physics Lists (PL) with the experimental flux measured in $\mathrm{n}_{-}$TOF-EAR1 [14]. These results indicate that all the models overestimate the integrated neutron flux, being QGSP_INCLXX_HPT the closest, just $12 \%$ above the experiment. Comparing the different PL's, the deviations in shape between the different hadronic models are clear at high energies (neutron kinectic energy above $10 \mathrm{MeV}$ ) and the choice of model also affects the magnitude of the integral flux at all energies. If we normalize to the experiment at $1 \mathrm{keV}$ to focus on the shape of the flux, we find a remarkably good reproduction of the experimental data from thermal to $10 \mathrm{MeV}$; above $10 \mathrm{MeV}$ all models
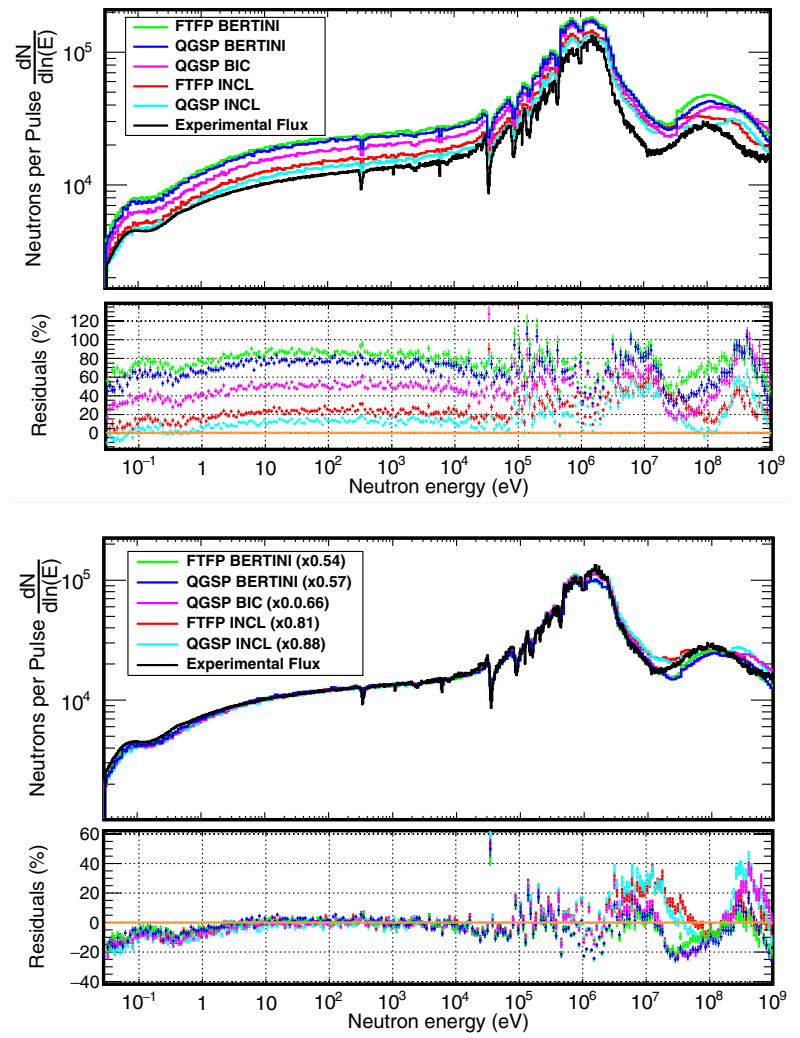

Figure 2. Top: average neutron flux per pulse at $n_{-}$TOF-EAR1 obtained with the different Geant4 Physics Lists (PL) compared to the experimental data (see [14]). Bottom: same comparison after normalizing the simulated results to the experimental data in the epithermal region to appreciate the good reproduction of the shape.

show discrepancies with respect to the measured neutron spectrum, being FTFP_BERT_HPT the physics list with smaller residuals (see Fig. 2, bottom panel). For more details of this work the reader is referred to Ref. [8].

\section{EAR2: Characteristics and prospects}

As in every new facility, a series of experimental campaigns are being carried out in order to determine the flux, spatial and energy distributions of the neutron beam, the associated backgrounds, the resolution broadening, and, in general, its measurement capabilities. However, not all these characteristics can be accurately measured, and certainly not in all the spatial positions and energy ranges of interest. In this context, Monte Carlo simulations become an essential tool for guiding the measurements, helping in their analysis, the interpretation of their results and, more importantly, for planning the upcoming physics experiments.

After benchmarking the different Geant4 PL with the experimental flux of EAR1, we extended our work to extract important results for $n_{-}$TOF-EAR2 that will be really helpful for feasibility studies, planning and analysis of the upcoming measurements. Figure 3 shows two relevant features predicted with these MC simulations. First, the $\gamma$-ray energy spectrum, shown in the top panel of Fig. 3, where two components are distinguished. The $\gamma$-rays produced via spallation reactions are emitted promptly with the arrival of the proton beam and arrive 

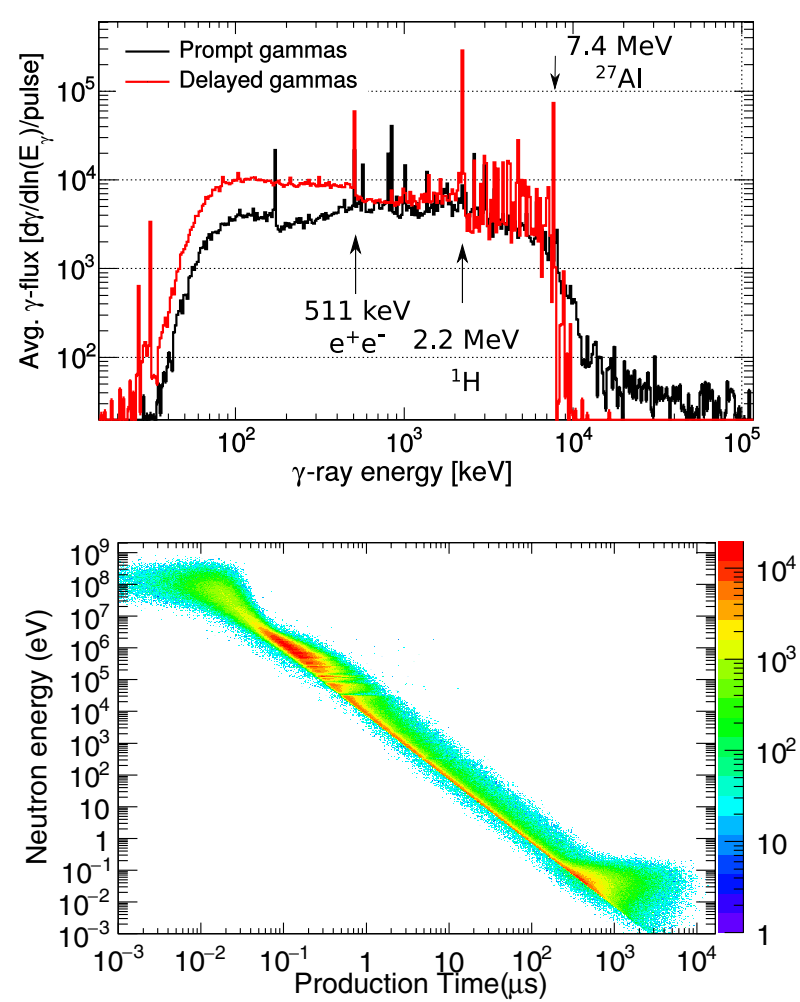

Figure 3. Top: energy distribution of the prompt and delayed $\gamma$-ray components in EAR2, including indications of the most prominent $\gamma$-rays from capture reactions and the annihilation peak. Bottom: resolution Function of the n_TOF-EAR2 neutron beam expressed in the form of neutron energy vs. production time (i.e. time of arrival at the scorer).

at EAR2 in less than $\sim 200 \mathrm{~ns}$. The $\gamma$-rays arriving after $\sim 200$ ns (thus delayed) are generated in capture reactions during the moderation process, up to a maximum delay of $10 \mathrm{~ms}$. The latter component, which presents some characteristic peaks (see Fig. 3), becomes a relevant source of background in capture experiments because it is difficult to distinguish these photons from the capture $\gamma$-rays of the investigated sample. Last, in the bottom panel of Fig. 3 we show the spread of the production times for a given neutron energy, the so-called Resolution Function, which is related to the spread of the original charged particle beam, the neutron production mechanism, and the neutron moderation. These effects introduce a broadening in the structures or resonances observed in time-of-flight experiments. A more detailed description of this work can be found in Ref. [9].

We acknowledge the n_TOF Collaboration. The research that led to these results has received funding from the EC FP7 Programme under the projects NEUTANDALUS (Grant No. 334315) and CHANDA (Grant No. 605203), and the Spanish Ministry of Economy and Competitiveness projects FPA2011-28770C3-02, FPA2013-45083-P and FPA2014- 53290-C2-2-P. The simulations have been performed at the computing cluster FISATOM, hosted at CICA (Seville, Spain), and of INFN-CNAF, University of Bologna; we thank the staff of both institutions for the operational maintenance of the machines.

\section{References}

[1] C. Guerrero et al. (The n_TOF Collaboration), Eur. Phys. J. A 49, 27 (2013)

[2] C. Weiss et al., Nucl Instrum. and Meth. A 799, 90-98 (2015)

[3] D.B. Pelowitz, ed., Los Alamos National Laboratory report, LA-CP-11-00438 (2011)

[4] A. Ferrari et al., CERN-2005-10,INFN/TC_05/11, SLAC-R-773 (2005)

[5] S. Agostinelli et al., Nucl. Instrum. and Meth. A 506, 250-303 (2003)

[6] J. Allison et al., IEEE Trans. Nucl. Sci. 53, 270-278 (2006)

[7] E. Mendoza et al., IEEE Trans. Nucl. Sci. 61, 2357 (2014)

[8] S. Lo Meo et al., Eur. Phys. J. A 51, 160 (2015)

[9] J. Lerendegui-Marco et al., Eur. Phys. J. A 52, 100 (2016)

[10] A. Boudard et al., Phys. Rev. C 87, 014606 (2013)

[11] D. Mancusi et al., Phys. Rev. C 90, 054602 (2014)

[12] A. Heikkinen et al., Bertini intra-nuclear cascade implementation in Geant4, in Computing in High Energy and Nuclear Physics, La Jolla, California, March 24-28, 2003

[13] G. Folger et al., Eur. Phys. J. A 21, 407-417 (2004)

[14] M. Barbagallo et al., (The n_TOF Collaboration), Eur. Phys. J. A 49, 156 (2013) 